\title{
Long-Term Symptoms Among Adults Tested for SARS-CoV-2 - United States, January 2020-April 2021
}

\begin{abstract}
Valentine Wanga, $\mathrm{PhD}^{1,2}$; Jennifer R. Chevinsky, MD ${ }^{1,2}$; Lina V. Dimitrov, MPH ${ }^{1,3}$; Megan E. Gerdes, $\mathrm{MPH}^{1,3}$; Geoffrey P. Whitfield, PhD ${ }^{4}$; Robert A. Bonacci, MD ${ }^{1,2}$; Miriam A.M. Nji, MD ${ }^{1}$; Alfonso C. Hernandez-Romieu, MD ${ }^{1,2}$; Jessica S. Rogers-Brown, PhD ${ }^{1}$; Tim McLeod, MPH ${ }^{1}$; Julie Rushmore, PhD, DVM ${ }^{1}$; Caitlyn Lutfy, $\mathrm{MPH}^{1}$; Dena Bushman, MSN, MPH ${ }^{1,2}$; Emilia Koumans, MD ${ }^{1}$; Sharon Saydah, PhD ${ }^{1}$; Alyson B. Goodman, $\mathrm{MD}^{1}$; Sallyann M. Coleman King, $\mathrm{MD}^{1}$; Brendan R. Jackson, $\mathrm{MD}^{1}$; Jennifer R. Cope, $\mathrm{MD}^{1}$
\end{abstract}

Long-term symptoms often associated with COVID-19 (post-COVID conditions or long COVID) are an emerging public health concern that is not well understood. Prevalence of post-COVID conditions has been reported among persons who have had COVID-19 (range $=5 \%-80 \%$ ), with differences possibly related to different study populations, case definitions, and data sources (1). Few studies of post-COVID conditions have comparisons with the general population of adults with negative test results for SARS-CoV-2, the virus that causes COVID-19, limiting ability to assess background symptom prevalence (1). CDC used a nonprobability-based Internet panel established by Porter Novelli Public Services* to administer a survey to a nationwide sample of U.S. adults aged $\geq 18$ years to compare the prevalence of long-term symptoms (those lasting $>4$ weeks since onset) among persons who self-reported ever receiving a positive SARS-CoV-2 test result with the prevalence of similar symptoms among persons who reported always receiving a negative test result. The weighted prevalence of ever testing positive for SARS-CoV-2 was $22.2 \%$ (95\% confidence interval $[\mathrm{CI}]=20.6 \%-23.8 \%)$. Approximately two thirds of respondents who had received a positive test result experienced longterm symptoms often associated with SARS-CoV-2 infection. Compared with respondents who received a negative test result, those who received a positive test result reported a significantly higher prevalence of any long-term symptom $(65.9 \%$ versus $42.9 \%)$, fatigue $(22.5 \%$ versus $12.0 \%)$, change in sense of smell or taste $(17.3 \%$ versus $1.7 \%)$, shortness of breath $(15.5 \%$ versus $5.2 \%)$, cough $(14.5 \%$ versus $4.9 \%)$, headache ( $13.8 \%$ versus $9.9 \%$ ), and persistence ( $>4$ weeks) of at least one initially occurring symptom (76.2\% versus $69.6 \%$ ). Compared with respondents who received a negative test result, a larger proportion of

\footnotetext{
${ }^{*}$ https://styles.porternovelli.com/pn-view-panels/
}

those who received a positive test result reported believing that receiving a COVID-19 vaccine made their long-term symptoms better $(28.7 \%$ versus $15.7 \%)$. Efforts to address post-COVID conditions should include helping health care professionals recognize the most common post-COVID conditions and optimize care for patients with persisting symptoms, including messaging on potential benefits of COVID-19 vaccination.

During April 9-23, 2021, Porter Novelli Public Services and ENGINE Insights ${ }^{\dagger}$ conducted a nonprobability-based Internet

${ }_{\dagger}$ https://engine-insights.com/product/caravan/

INSIDE

1242 Using Wastewater Surveillance Data to Support the COVID-19 Response - United States, 2020-2021

1245 SARS-CoV-2 Transmission to Masked and Unmasked Close Contacts of University Students with COVID-19 - St. Louis, Missouri, January-May 2021

1249 Trends in COVID-19 Cases, Emergency Department Visits, and Hospital Admissions Among Children and Adolescents Aged 0-17 Years - United States, August 2020-August 2021

1255 Hospitalizations Associated with COVID-19 Among Children and Adolescents - COVID-NET, 14 States, March 1, 2020-August 14, 2021

1261 Notes from the Field:Tuberculosis Outbreak Linked to a Contaminated Bone Graft Product Used in Spinal Surgery — Delaware, March-June 2021

1264 QuickStats

Continuing Education examination available at https://www.cdc.gov/mmwr/mmwr_continuingEducation.html

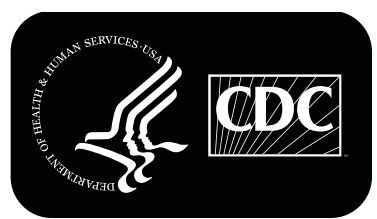

U.S. Department of Health and Human Services Centers for Disease Control and Prevention 
panel survey among 6,021 noninstitutionalized U.S. adults aged $\geq 18$ years via the Lucid platform. ${ }^{\complement}$ Quota sampling and statistical weighting were used to align the sample with U.S. population distributions by sex, age group, U.S. Census region, race and ethnicity, and education. Respondents self-reported ever having received a positive SARS-CoV-2 test result (698), always receiving a negative test result $(2,437)$, or never having been tested for SARS-CoV-2 (2,750); only deidentified respondents who reported having received either a positive or a negative test result were included in this analysis. Respondents who received a negative test were selected to establish the prevalence of post-COVID symptoms in a population that did not receive a COVID-19 diagnosis. Assessment of initial symptoms, including symptoms that might have commenced before testing, ' was conducted by asking respondents who received a positive test result, "During the month of your first positive COVID-19 test, which, if any, of the following symptoms did you experience?" followed by a list of symptoms. Respondents who received a negative test result were asked, "Since January 2020, which, if any, of the following symptoms have you experienced?" Long-term symptoms

\footnotetext{
$\$$ https://luc.id/marketplace/

Change in mood; change in smell or taste; chest pain or pressure; cough; diarrhea; difficulty thinking clearly, concentrating, forgetfulness, memory loss, or "brain fog"; fatigue, tired, or weakness; fever or chills; hair loss; headache; joint or muscle pain; nausea or vomiting; palpitations (heart racing or pounding); postexertional malaise (worsening of symptoms after even minor physical, mental, or emotional exertion); problems sleeping; shortness of breath or breathlessness; sore throat; stomach pain; or other symptom.
}

were assessed by asking those who received a positive test result, "Which, if any, of your symptoms lasted longer than 4 weeks after your first positive COVID-19 test?"; those who received a negative test result were asked, "Which, if any, of your symptoms lasted longer than 4 weeks since you first experienced the symptoms?" Respondents were asked about health care use and receipt of $\geq 1$ dose of a COVID-19 vaccine. Vaccine impact related to symptoms was assessed by asking respondents** how receiving a COVID-19 vaccination affected their long-term symptoms (those lasting $\geq 4$ weeks). ${ }^{\dagger \dagger}$

Point estimates and 95\% CIs were calculated, overall and by demographic characteristics (age group, sex, marital status, highest educational attainment, employment, 2020 household income, race and ethnicity, U.S. Census region, and community type ${ }^{\mathbb{S}) \text {. }}$

\footnotetext{
** Among those who experienced long-term symptoms and reported receiving $\geq 1$ dose of a COVID-19 vaccine (100 respondents who received a positive test result and 285 respondents who received a negative test result).

t† Respondents were instructed, "Please select only one answer and do not count side effects (symptoms that you had in the first 7 days after getting a COVID-19 vaccine)." Responses included, "Made my symptoms a lot better," "Made my symptoms somewhat better," "Made my symptoms a little better," "Did not affect my symptoms at all," "Made my symptoms a little worse," "Made my symptoms somewhat worse," "Made my symptoms a lot worse," "Made some of my symptoms better and some worse," and "Not applicable: My symptoms were gone before I got the vaccine." Because of small cell sizes, those reporting that receiving a vaccine made their symptoms a lot, somewhat, or a little better were grouped under "Getting vaccine made symptoms better" and those reporting that receiving vaccine made their symptoms a lot, somewhat, or a little worse were grouped under "Getting vaccine made symptoms worse."

$\$ \$$ Urban, suburban, or rural.
}

The MMWR series of publications is published by the Center for Surveillance, Epidemiology, and Laboratory Services, Centers for Disease Control and Prevention (CDC), U.S. Department of Health and Human Services, Atlanta, GA 30329-4027.

Suggested citation: [Author names; first three, then et al., if more than six.] [Report title]. MMWR Morb Mortal Wkly Rep 2021;70:[inclusive page numbers]

\section{Centers for Disease Control and Prevention Rochelle P. Walensky, MD, MPH, Director \\ Debra Houry, MD, MPH, Acting Principal Deputy Director \\ Daniel B. Jernigan, MD, MPH, Deputy Director for Public Health Science and Surveillance \\ Rebecca Bunnell, PhD, MEd, Director, Office of Science \\ Jennifer Layden, MD, PhD, Deputy Director, Office of Science \\ Michael F. Iademarco, MD, MPH, Director, Center for Surveillance, Epidemiology, and Laboratory Services}

MMWR Editorial and Production Staff (Weekly)

Charlotte K. Kent, $\mathrm{PhD}$, MPH, Editor in Chief Jacqueline Gindler, MD, Editor

Brian A. King, PhD, MPH, Guest Science Editor

Paul Z. Siegel, MD, MPH, Associate Editor

Mary Dott, MD, MPH, Online Editor

Terisa F. Rutledge, Managing Editor

Teresa M. Hood, MS, Lead Technical Writer-Editor

Leigh Berdon, Glenn Damon, Soumya Dunworth, PhD, Srila Sen, MA, Stacy Simon, MA,

Jeffrey D. Sokolow, MA, Morgan Thompson, Technical Writer-Editors

Matthew L. Boulton, MD, MPH

Carolyn Brooks, ScD, MA

Jay C. Butler, MD

Virginia A. Caine, MD

Jonathan E. Fielding, MD, MPH, MBA

David W. Fleming, MD
Martha F. Boyd, Lead Visual Information Specialist

Alexander J. Gottardy, Maureen A. Leahy,

Julia C. Martinroe, Stephen R. Spriggs,

Brian Taitt, Tong Yang

Visual Information Specialists

Quang M. Doan, MBA, Phyllis H. King,

Terraye M. Starr, Moua Yang,

Information Technology Specialists

MMWR Editorial Board

Timothy F. Jones, MD, Chairman

William E. Halperin, MD, DrPH, MPH

Jewel Mullen, MD, MPH, MPA

Jeff Niederdeppe, $\mathrm{PhD}$

Celeste Philip, MD, MPH

Patricia Quinlisk, MD, MPH

Patrick L. Remington, MD, MPH
Ian Branam, MA

Acting Lead Health Communication Specialist

Shelton Bartley, MPH,

Lowery Johnson, Amanda Ray,

Jacqueline N. Sanchez, MS,

Health Communication Specialists

Will Yang, MA,

Visual Information Specialist 
Comparisons of demographic characteristics and symptoms were performed using chi-square tests; p-values $<0.05$ were considered statistically significant. All analyses were conducted using SAS (version 9.4; SAS Institute) and were weighted by sex, age group, region, race and ethnicity, and education. This activity was reviewed by $\mathrm{CDC}$ and was conducted consistent with applicable federal law and CDC policy.99

Among the 3,135 adults who reported having been tested for SARS-CoV-2 since January 2020, the weighted prevalence of ever receiving a positive test result was $22.2 \%$ (Table 1 ). Compared with respondents who received a negative test result $(2,437)$, those who received a positive test result $(698)$ were younger (median age $=39.3$ years versus 45.3 years), and a higher proportion were working $(70.5 \%$ versus $61.6 \%)$, had higher household income $(50.8 \%$ versus $43.9 \%$ made $\geq \$ 60,000)$, and lived in an urban community ( $43.8 \%$ versus $37.6 \%$ ).

Overall, $603(86.5 \%)$ respondents who received a positive test result and 1,526 (61.7\%) of those who received a negative test result reported any initial symptoms. Among respondents who reported an initial symptom, more respondents who received a positive test result $(76.2 \%)$ than those who received a negative test result $(69.6 \%)$ reported persistence ( $>4$ weeks) of at least one symptom ( $\mathrm{p}=0.005)$ (Supplementary Table, https://stacks.cdc.gov/view/cdc/108815). Hair loss (58.3\%), cognitive dysfunction ${ }^{* * *}(55.5 \%)$, shortness of breath $(52.8 \%)$, and postexertional malaise ${ }^{\dagger \dagger \dagger}(49.6 \%)$ persisted for $\geq 50 \%$ of those who received a positive test result and initially reported these symptoms; other symptoms, such as fatigue (48.4\%), change in smell or taste $(46.4 \%)$, cough $(36.2 \%)$, and headache $(31.1 \%)$ persisted for $<50 \%$.

A higher proportion of respondents who received a positive test result than those who received a negative test result reported any long-term symptoms $(65.9 \%$ versus $42.9 \%$; $\mathrm{p}<0.05$ ) (Table 2). The most common symptoms were fatigue (22.5\% versus $12.0 \%)$, change in smell or taste ( $17.3 \%$ versus $1.7 \%)$, shortness of breath (15.5\% versus $5.2 \%)$, cough $(14.5 \%$ versus $4.9 \%)$, and headache (13.8\% versus $9.9 \%)$. Among only respondents who reported any long-term symptoms, the most common symptoms among those who received a positive test result compared with those who received a negative test result were fatigue $(34.2 \%$ versus $28.0 \%)$, change in smell or taste $(26.2 \%$ versus $3.9 \%)$, shortness of breath $(23.6 \%$ versus $12.1 \%)$, cough $(22.0 \%$ versus $11.5 \%)$, and headache $(20.9 \%$ versus $23.0 \%$ ) (all $\mathrm{p}<0.05$ except for headache).

\footnotetext{
9945 C.F.R. part 46; 21 C.F.R. part 56; 42 U.S.C. Sect. 241(d); 5 U.S.C. Sect. 552a; 44 U.S.C. Sect. 3501 et seq.

*** Cognitive dysfunction refers to difficulty thinking clearly, concentrating, forgetfulness, memory loss, or "brain fog."

$\dagger \dagger \dagger$ Worsening of symptoms after even minor physical, mental, or emotional exertion.
}

A larger proportion of respondents who received a positive test result than those who received a negative test result reported seeing a health care professional $(54.1 \%$ versus $42.5 \%$; $\mathrm{p}<0.001)$ or going to urgent or emergency care $(19.5 \%$ versus $14.0 \%, \mathrm{p}=0.008)$ for symptoms when they first occurred; rates of hospitalization were similar (10.4\% versus $9.3 \%)$ (Table 3). Among those reporting any long-term symptoms, fewer respondents who received a positive test result than those who received a negative test result reported seeing a health care professional for long-term symptoms at least once $(24.7 \%$ versus $35.8 \%$ ) or more than once (17.6\% versus $27.9 \%)$.

Fewer respondents who received a positive test result than those who received a negative test result reported receiving $\geq 1$ dose of a COVID-19 vaccine (28.3\% versus $39.4 \%$ ). Among those who ever experienced any long-term symptoms, more respondents who received a positive test result than those who received a negative test result reported that having longterm symptoms motivated them to receive or consider receiving a COVID-19 vaccine (11.0\% versus $7.0 \%)$ and believed that receiving the vaccine made their long-term symptoms better $(28.7 \%$ versus $15.7 \% ; \mathrm{p}=0.023)$, or that their symptoms were gone before receiving the vaccine ( $28.4 \%$ versus $13.1 \%$ ). A similar percentage of respondents who received a positive test result $(16.1 \%)$ and those who received a negative test result $(11.2 \%)$ reported that receiving the vaccine made their long-term symptoms worse ( $p=0.271$ ), whereas $26.4 \%$ of respondents who received a positive test result and $59.2 \%$ of those who received a negative test result believed that receiving a vaccine did not affect their symptoms $(\mathrm{p}<0.001)$.

\section{Discussion}

In this convenience sample of U.S. adults, the prevalence of long-term symptoms often associated with SARS-CoV-2 infection was higher among respondents who ever received a positive test result than among those who always received a negative test result, and symptoms in these persons tended to persist for $>4$ weeks. Previous studies have found that nonhospitalized persons with SARS-CoV-2 infection have higher prevalence of some long-term symptoms or conditions than nonhospitalized persons with negative SARS-CoV-2 test results (2-5). Similarly, in this investigation, more respondents who received a positive test result $(65.9 \%)$ than those who received a negative test result $(42.9 \%)$ experienced any long-term symptoms, and approximately one half of these symptoms were more likely to be reported among those who received a positive test result.

Early data on post-COVID conditions primarily came from hospitalized cohorts $(1,6)$; more recent reports describe postCOVID conditions among nonhospitalized, asymptomatic, or mildly ill patients $(1,7)$. The prevalence of the most common long-term symptoms among respondents who received 
TABLE 1. Demographic characteristics of respondents aged $\geq 18$ years who received at least one positive SARS-CoV-2 test result or only negative SARS-CoV-2 test results since January $2020(\mathrm{~N}=3,135)$, by selected characteristics - Porter Novelli Internet survey, United States, April 2021

\begin{tabular}{|c|c|c|c|}
\hline \multirow[b]{2}{*}{ Characteristic } & \multicolumn{2}{|c|}{ Testing status, weighted $\%(95 \% \mathrm{CI})$} & \multirow[b]{2}{*}{ p-value ${ }^{\dagger}$} \\
\hline & $\begin{array}{l}\text { At least one positive } \\
\text { SARS-CoV-2 test result } \\
(n=698)^{*}\end{array}$ & $\begin{array}{c}\text { All negative } \\
\text { SARS-CoV-2 test results } \\
(n=2,437)^{*}\end{array}$ & \\
\hline Overall & $22.2(20.6-23.8)$ & $77.8(76.2-79.4)$ & - \\
\hline \multicolumn{4}{|l|}{ Age group, yrs } \\
\hline $18-29$ & $26.3(22.8-29.7)$ & $23.2(21.4-24.9)$ & $<0.001$ \\
\hline $30-39$ & $25.4(22.0-28.8)$ & $19.0(17.4-20.6)$ & $<0.001$ \\
\hline $40-49$ & $18.6(15.1-22.1)$ & $16.4(14.7-18.2)$ & $<0.001$ \\
\hline $50-59$ & $15.0(11.8-18.2)$ & $16.6(14.8-18.4)$ & $<0.001$ \\
\hline $60-69$ & $10.3(7.8-12.8)$ & $16.4(14.8-18.0)$ & $<0.001$ \\
\hline$\geq 70$ & $4.4(2.8-6.0)$ & $8.4(7.2-9.5)$ & $<0.001$ \\
\hline \multicolumn{4}{|l|}{ Sex } \\
\hline Male & $51.5(47.4-55.7)$ & $48.5(46.3-50.7)$ & 0.204 \\
\hline Female & $48.5(44.3-52.6)$ & $51.5(49.3-53.7)$ & 0.204 \\
\hline \multicolumn{4}{|l|}{ Marital status } \\
\hline Married & $54.0(49.9-58.1)$ & $49.2(47.0-51.4)$ & 0.197 \\
\hline Living with a partner & $10.2(7.7-12.6)$ & $10.2(8.8-11.6)$ & 0.197 \\
\hline Single and never been married & $22.2(18.7-25.6)$ & $24.4(22.6-26.3)$ & 0.197 \\
\hline Other $\S$ & $13.6(10.6-16.6)$ & $16.1(14.5-17.8)$ & 0.197 \\
\hline \multicolumn{4}{|l|}{ Highest level of education completed } \\
\hline Some high school or less & $4.7(2.8-6.6)$ & $5.2(4.1-6.3)$ & 0.545 \\
\hline High school graduate/some college & $50.7(46.6-54.8)$ & $49.2(47.0-51.3)$ & 0.545 \\
\hline 2-year college/technical school & $7.8(5.7-10.0)$ & $8.5(7.3-9.8)$ & 0.545 \\
\hline 4-year college/some postgraduate education & $19.6(16.6-22.5)$ & $21.7(20.1-23.3)$ & 0.545 \\
\hline Postgraduate degree & $17.2(14.4-19.9)$ & $15.4(14.1-16.8)$ & 0.545 \\
\hline \multicolumn{4}{|l|}{ Employment status? } \\
\hline Employed & $70.5(66.6-74.3)$ & $61.6(59.5-63.8)$ & $<0.001$ \\
\hline Unemployed & $17.8(14.5-21.1)$ & $19.8(17.9-21.6)$ & $<0.001$ \\
\hline Retired & $11.7(9.0-14.4)$ & $18.6(16.9-20.3)$ & $<0.001$ \\
\hline \multicolumn{4}{|l|}{ Household income in 2020, USD } \\
\hline$<25,000$ & $14.5(11.4-17.5)$ & $20.4(18.5-22.2)$ & 0.016 \\
\hline $25,000-49,999$ & $28.2(24.4-32.0)$ & $27.8(25.8-29.9)$ & 0.016 \\
\hline $50,000-74,999$ & $17.2(14.0-20.4)$ & $17.3(15.7-19.0)$ & 0.016 \\
\hline $75,000-99,999$ & $12.0(9.5-14.5)$ & $11.3(9.9-12.6)$ & 0.016 \\
\hline $100,000-149,999$ & $16.3(13.5-19.2)$ & $14.4(13.0-15.7)$ & 0.016 \\
\hline$\geq 150,000$ & $11.8(9.2-14.3)$ & $8.9(7.8-10.0)$ & 0.016 \\
\hline \multicolumn{4}{|l|}{ Race/Ethnicity } \\
\hline White, non-Hispanic & $59.8(55.5-64.0)$ & $62.0(59.7-64.2)$ & 0.190 \\
\hline Black or African-American, non-Hispanic & $12.4(9.6-15.3)$ & $12.3(10.8-13.9)$ & 0.190 \\
\hline Other, ${ }^{* *}$ non-Hispanic & $6.2(4.1-8.4)$ & $8.2(6.9-9.5)$ & 0.190 \\
\hline Hispanic & $21.6(17.6-25.5)$ & $17.5(15.6-19.4)$ & 0.190 \\
\hline \multicolumn{4}{|l|}{ U.S. Census region ${ }^{\dagger+}$} \\
\hline Northeast & $21.5(18.1-24.9)$ & $19.0(17.3-20.6)$ & 0.156 \\
\hline Midwest & $21.1(17.7-24.5)$ & $19.5(17.7-21.2)$ & 0.156 \\
\hline South & $38.1(34.1-42.1)$ & $38.4(36.3-40.5)$ & 0.156 \\
\hline West & $19.3(16.1-22.5)$ & $23.1(21.3-25.0)$ & 0.156 \\
\hline \multicolumn{4}{|l|}{ Community type } \\
\hline Urban & $43.8(39.8-47.9)$ & $37.6(35.5-39.7)$ & 0.028 \\
\hline Suburban & $39.8(35.7-43.9)$ & $44.1(41.9-46.3)$ & 0.028 \\
\hline Rural & $16.4(13.3-19.5)$ & $18.3(16.6-20.0)$ & 0.028 \\
\hline
\end{tabular}

Abbreviations: $\mathrm{Cl}=$ confidence interval; $\mathrm{USD}=$ U.S. dollars.

* Unweighted number of persons who received positive or negative SARS-CoV-2 test results.

${ }^{\dagger} p$-value for weighted Wald chi-square test; all $p$-values $<0.05$ indicate significant differences.

$\S$ Other marital status includes separated, divorced, and widowed.

" Employed includes full-time or part-time work and self-employment; unemployed includes students, homemakers, and those who were not employed currently or were unable to work.

** Other race/ethnicity includes Native American or Alaskan Native, Asian, and other (unspecified).

${ }^{++}$Northeast: Connecticut, Maine, Massachusetts, New Hampshire, New Jersey, New York, Pennsylvania, Rhode Island, and Vermont; Midwest: Illinois, Indiana, lowa, Kansas, Michigan, Minnesota, Missouri, Nebraska, North Dakota, Ohio, South Dakota, and Wisconsin; South: Alabama, Arkansas, Delaware, District of Columbia, Florida, Georgia, Kentucky, Louisiana, Maryland, Mississippi, North Carolina, Oklahoma, South Carolina, Tennessee, Texas, Virginia, and West Virginia; West: Alaska, Arizona, California, Colorado, Hawaii, Idaho, Montana, Nevada, New Mexico, Oregon, Utah, Washington, and Wyoming. 
TABLE 2. Prevalence of symptoms lasting $>4$ weeks among respondents aged $\geq 18$ years who received at least one positive SARS-CoV-2 test result or only negative SARS-CoV-2 test results since January 2020 - Porter Novelli Internet survey, United States, April 2021

SARS-CoV-2 test result, weighted $\%(95 \% \mathrm{Cl})$

\begin{tabular}{|c|c|c|c|c|}
\hline \multirow[b]{2}{*}{ Symptom } & \multicolumn{2}{|c|}{$\begin{array}{c}\text { Symptom prevalence } \\
\text { among all persons receiving testing }\end{array}$} & \multicolumn{2}{|c|}{$\begin{array}{l}\text { Symptom prevalence among persons receiving testing } \\
\text { who reported a symptom lasting }>4 \text { weeks since onset }\end{array}$} \\
\hline & $\begin{array}{l}\text { Respondents who } \\
\text { received a } \\
\text { positive test result } \\
(n=698)^{*}\end{array}$ & $\begin{array}{l}\text { Respondents who } \\
\text { received a } \\
\text { negative test result } \\
(n=2,437)^{*}\end{array}$ & $\begin{array}{l}\text { Respondents who } \\
\text { received a } \\
\text { positive test result } \\
(\mathrm{n}=465)^{*}\end{array}$ & $\begin{array}{l}\text { Respondents who } \\
\text { received a } \\
\text { negative test result } \\
(n=1,058)^{*}\end{array}$ \\
\hline Any symptom & $65.9^{\dagger}(61.9-69.8)$ & $42.9(40.8-45.1)$ & - & - \\
\hline 1 symptom only & $27.2^{\dagger}(23.7-30.8)$ & $18.7(17.0-20.4)$ & $41.4(36.5-46.3)$ & $43.6(40.3-46.9)$ \\
\hline 2 symptoms & $14.0^{\dagger}(11.1-16.8)$ & $9.5(8.2-10.7)$ & $21.2(17.1-25.3)$ & $22.1(19.3-24.8)$ \\
\hline$\geq 3$ symptoms & $24.7^{\dagger}(21.0-28.3)$ & $14.7(13.2-16.3)$ & $37.4(32.5-42.4)$ & $34.3(31.1-37.5)$ \\
\hline Fatigue/Tired/Weakness & $22.5^{+}(19.0-26.1)$ & $12.0(10.6-13.4)$ & $34.2^{\dagger}(29.3-39.1)$ & $28.0(25.0-31.0)$ \\
\hline Change in smell or taste & $17.3^{\dagger}(14.1-20.4)$ & $1.7(1.1-2.3)$ & $26.2^{\dagger}(21.8-30.7)$ & $3.9(2.6-5.3)$ \\
\hline Shortness of breath or breathlessness & $15.5^{\dagger}(12.4-18.7)$ & $5.2(4.2-6.2)$ & $23.6^{\dagger}(19.1-28.1)$ & $12.1(9.9-14.2)$ \\
\hline Cough & $14.5^{\dagger}(11.6-17.4)$ & $4.9(4.0-5.9)$ & $22.0^{\dagger}(17.8-26.2)$ & $11.5(9.4-13.6)$ \\
\hline Headache & $13.8^{\dagger}(10.9-16.7)$ & $9.9(8.6-11.2)$ & $20.9(16.7-25.1)$ & $23.0(20.2-25.8)$ \\
\hline Problems sleeping & $12.0^{\dagger}(9.3-14.7)$ & $16.5(14.8-18.1)$ & $18.1^{\dagger}(14.2-22.1)$ & $38.3(35.1-41.6)$ \\
\hline Joint or muscle pain & $11.1(8.4-13.9)$ & $12.4(10.9-13.9)$ & $16.9^{\dagger}(12.9-20.9)$ & $28.9(25.8-32.0)$ \\
\hline Cognitive dysfunction $\$$ & $10.2^{\dagger}(7.7-12.8)$ & $7.3(6.1-8.4)$ & $15.5(11.8-19.3)$ & $16.9(14.4-19.4)$ \\
\hline Chest pain or pressure & $7.3^{\dagger}(5.2-9.4)$ & $2.3(1.6-2.9)$ & $11.0^{\dagger}(7.9-14.2)$ & $5.3(3.7-6.8)$ \\
\hline Change in mood & $6.6(4.6-8.7)$ & $8.8(7.6-10.0)$ & $10.1^{\dagger}(7.1-13.1)$ & $20.6(17.9-23.2)$ \\
\hline Postexertional malaise & $6.1^{\dagger}(4.1-8.0)$ & $2.4(1.7-3.0)$ & $9.2^{\dagger}(6.3-12.2)$ & $5.5(3.9-7.0)$ \\
\hline Stomach pain & $5.8(3.9-7.7)$ & $5.1(4.1-6.1)$ & $8.9(6.0-11.7)$ & $11.9(9.7-14.1)$ \\
\hline Hair loss & $5.6(3.7-7.5)$ & $4.1(3.3-5.0)$ & $8.5(5.6-11.3)$ & $9.7(7.6-11.7)$ \\
\hline Diarrhea & $5.3(3.3-7.2)$ & $3.3(2.6-4.1)$ & $8.0(5.0-10.9)$ & $7.8(6.0-9.5)$ \\
\hline Sore throat & $4.9^{\dagger}(3.1-6.8)$ & $1.7(1.1-2.2)$ & $7.5^{\dagger}(4.7-10.3)$ & $3.9(2.7-5.1)$ \\
\hline Fever or chills & $4.9^{\dagger}(3.0-6.8)$ & $1.9(1.4-2.5)$ & $7.5(4.7-10.3)$ & $4.5(3.2-5.8)$ \\
\hline Palpitations (heart racing or pounding) & $4.5(2.7-6.3)$ & $2.5(1.9-3.2)$ & $6.8(4.1-9.5)$ & $5.9(4.3-7.5)$ \\
\hline Nausea/Vomiting & $4.1^{\dagger}(2.5-5.8)$ & $1.9(1.3-2.4)$ & $6.3(3.8-8.8)$ & $4.3(3.0-5.7)$ \\
\hline Other symptom & $1.3(0.3-2.2)^{* *}$ & $1.0(0.6-1.5)$ & $2.0(0.5-3.4)^{* *}$ & $2.4(1.4-3.4)$ \\
\hline
\end{tabular}

Abbreviation: $\mathrm{Cl}=$ confidence interval.

* Unweighted number of persons who received at least one positive or only negative SARS-CoV-2 test results.

${ }^{\dagger} p$-value for weighted Wald chi-square test $<0.05$, indicating significant differences between those receiving a positive test result and those receiving a negative SARS-CoV- 2 test result.

$\S$ Difficulty thinking clearly, concentrating, forgetfulness, memory loss, or "brain fog."

" Worsening of symptoms after even minor physical, mental, or emotional exertion.

** Estimate is unstable; relative standard error is $>30 \%$.

a positive test result in this investigation was similar to that in earlier studies $(1,8)$. Many studies on post-COVID conditions lack comparisons with the general population of adults with negative test results for SARS-CoV-2; however, this investigation included a comparison group, allowing for assessment of background symptom frequencies. Estimating population-level frequency of specific long-term symptoms among the general population and patients infected with SARS-CoV-2 could help health care professionals better understand the types and prevalences of symptoms their patients might experience and could help guide health systems in preparing care management strategies for patients with post-COVID conditions.

Among respondents who initially reported symptoms during the month of their first positive test results, $>75 \%$ reported persistence of any symptoms $>4$ weeks, with hair loss, cognitive dysfunction, shortness of breath, and postexertional malaise persisting in approximately one half of respondents. This finding is consistent with findings from other studies reported in a systematic review (1) and provides patient-level perspective on long-term symptoms associated with COVID-19; taken together, these studies highlight the importance of continued monitoring and clinical care for long-term symptoms among patients who have these symptoms early in the course of their illness.

With the increasing availability of COVID-19 vaccines, how vaccination affects post-COVID conditions remains unclear. Compared with respondents who received a negative test result, a higher proportion of those who received a positive test result believed that receiving a COVID-19 vaccine made their long-term symptoms better, and no difference was found in reported beliefs that receiving a vaccine made long-term symptoms worse. Early findings indicate that vaccination is not associated with worsening of post-COVID conditions ${ }^{\$ \$ \$}$ (9). However, because no data were collected on the trajectory of long-term symptoms in persons who had not been vaccinated, whether any of the observed changes in symptoms are attributable to vaccination is uncertain. More data are needed to fully

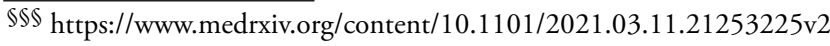


TABLE 3. Frequency of health care use, vaccination, and reported vaccine effects on symptoms lasting $>4$ weeks among respondents aged $\geq 18$ years who received at least one positive SARS-CoV-2 test result or only negative SARS-CoV-2 test results since January 2020 - Porter Novelli Internet survey, United States, April 2021

\begin{tabular}{|c|c|c|c|}
\hline \multirow[b]{2}{*}{ Item } & \multicolumn{2}{|c|}{ Weighted \% $(95 \% \mathrm{Cl})$} & \multirow[b]{2}{*}{ p-value* } \\
\hline & $\begin{array}{l}\text { At least one positive } \\
\text { SARS-CoV-2 test result }\end{array}$ & $\begin{array}{l}\text { All negative } \\
\text { SARS-CoV-2 test results }\end{array}$ & \\
\hline Health care utilization among those with any initial symptom & $603^{\dagger}$ & $1,526^{\dagger}$ & N/A \\
\hline Saw health care professional for symptoms & $54.1(49.7-58.6)$ & $42.5(39.8-45.2)$ & $<0.001$ \\
\hline Went to urgent or emergency care for symptoms & $19.5(16.0-23.0)$ & $14.0(12.1-15.9)$ & 0.008 \\
\hline Hospitalized for symptoms & $10.4(7.8-13.0)$ & $9.3(7.6-11.0)$ & 0.492 \\
\hline $\begin{array}{l}\text { Health care utilization among those with symptoms lasting }>4 \text { wks } \\
\text { (long-term symptoms) }\end{array}$ & $465^{\dagger}$ & $1,058^{\dagger}$ & N/A \\
\hline Saw health care professional for long-term symptoms & $24.7(20.5-28.9)$ & $35.8(32.6-39.0)$ & $<0.001$ \\
\hline Saw health care professional more than once for long-term symptoms & $17.6(13.7-21.5)$ & $27.9(24.8-30.9)$ & $<0.001$ \\
\hline COVID-19 vaccination status & $698^{\dagger}$ & $2,437^{\dagger}$ & N/A \\
\hline Received at least 1 dose of vaccine ${ }^{\S}$ & $28.3(24.5-32.0)$ & $39.4(37.3-41.5)$ & $<0.001$ \\
\hline $\begin{array}{l}\text { Having long-term symptoms was a motivator to receive or consider } \\
\text { receiving vaccine }\end{array}$ & $11.0(8.0-14.0)$ & $7.0(5.3-8.7)$ & 0.023 \\
\hline Reported vaccination effects on long-term symptoms & $100^{\dagger}$ & $285^{\dagger}$ & N/A \\
\hline Vaccine made symptoms better** & $28.7(18.6-38.7)$ & $15.7(11.3-20.0)$ & 0.023 \\
\hline Vaccine did not affect symptoms at all & $26.4(16.7-36.0)$ & $59.2(53.1-65.4)$ & $<0.001$ \\
\hline Vaccine made symptoms worse ${ }^{t \dagger}$ & $16.1(8.4-23.7)$ & $11.2(6.9-15.4)$ & 0.271 \\
\hline Symptoms were gone before receiving vaccine & $28.4(18.4-38.5)$ & $13.1(8.9-17.3)$ & 0.007 \\
\hline
\end{tabular}

Abbreviations: $\mathrm{Cl}=$ confidence interval; $\mathrm{N} / \mathrm{A}=$ not applicable.

* $p$-value for weighted Wald chi-square test; all p-values $<0.05$ indicate significant differences.

+ Unweighted number of persons who received positive or negative SARS-CoV-2 test results.

$\S 189$ of 698 respondents who received a positive test result and 961 of 2,437 respondents who received a negative test result reported receiving 1 dose of vaccine.

" Respondents who ever experienced a long-term symptom and received at least 1 vaccine dose.

** Includes those reporting receiving a vaccine made symptoms a lot better, somewhat better, or a little better.

${ }^{+\dagger}$ Includes those reporting receiving a vaccine made symptoms a little worse, somewhat worse, or a lot worse.

understand the effects of COVID-19 vaccines on persons with post-COVID conditions.

The findings in this report are subject to at least six limitations. First, the study used a nonprobability-based sample, which limits its generalizability. Second, responses were selfreported and thus subject to recall bias. Third, new symptoms occurring after the month when the first positive COVID-19 test result was received among those who received a positive test result were not assessed, and the reported symptoms could not be linked directly to SARS-CoV-2. Fourth, because the survey did not ask about symptom duration or severity, differences in duration or severity of long-term symptoms in respondents who received a positive rather than a negative test result could not be assessed. Fifth, respondents who always received a negative test result generally had a longer period in which to report symptoms, potentially inflating prevalence of their health care use and long-term symptoms. Finally, this study could not assess validity of SARS-CoV-2 tests, and some false-positive or false-negative test results might have resulted in misclassification of some respondents.

These findings can help guide public health preparedness efforts, resource needs for care and management of persons with post-COVID conditions, and communication about experiences with vaccination. The findings can also aid efforts to address post-COVID conditions, including helping health

\section{Summary}

What is already known about this topic?

Long-term symptoms associated with COVID-19 represent an emerging public health concern.

What is added by this report?

In a nonprobability-based sample of U.S. adults tested for SARS-CoV-2, symptoms often associated with SARS-CoV-2 infection were common; $65.9 \%$ of respondents whose SARS-CoV-2 test results were positive reported symptoms lasting $>4$ weeks compared with $42.9 \%$ of those whose test results were negative. More persons who received positive test results (76.2\%) reported persistence ( $>4$ weeks) of at least one initially occurring symptom compared with those whose test results were negative $(69.6 \%)$.

What are the implications for public health practice?

These findings can aid efforts to address post-COVID conditions and messaging on potential benefits of vaccination.

care professionals recognize the most common symptoms and optimize care for patients whose symptoms persist. Future research to assess long-term symptoms and risk factors, including disease severity, disease duration, and sociodemographic characteristics, will be important to help guide current and future health care services. 
Corresponding author: Valentine Wanga, qdy0@cdc.gov.

${ }^{1}$ CDC COVID-19 Response Team; ${ }^{2}$ Epidemic Intelligence Service, CDC; ${ }^{3}$ Oak Ridge Institute for Science and Education, Oak Ridge, Tennessee;

${ }^{4}$ National Center for Chronic Disease Prevention and Health Promotion, CDC.

All authors have completed and submitted the International Committee of Medical Journal Editors form for disclosure of potential conflicts of interest. No potential conflicts of interest were disclosed.

\section{References}

1. Cabrera Martimbianco AL, Pacheco RL, Bagattini ÂM, Riera R. Frequency, signs and symptoms, and criteria adopted for long COVID-19: a systematic review. Int J Clin Pract 2021;e14357. Epub May 11, 2021. PMID:33977626

2. Office for National Statistics. Prevalence of ongoing symptoms following coronavirus (COVID-19) infection in the UK: 1 April 2021. London, UK: Office for National Statistics; 2021. https://www. ons.gov.uk/peoplepopulationandcommunity/healthandsocialcare/ conditionsanddiseases/bulletins/fongoingsymptomsfollowingcoronavir uscovid19infectionintheuk/1april2021

3. Chevinsky JR, Tao G, Lavery AM, et al. Late conditions diagnosed 1-4 months following an initial COVID-19 encounter: a matched cohort study using inpatient and outpatient administrative data-United States, March 1-June 30, 2020. Clin Infect Dis 202173(Suppl 1):S5-16. https:// doi.org/10.1093/cid/ciab338
4. Lund LC, Hallas J, Nielsen H, et al. Post-acute effects of SARS-CoV-2 infection in individuals not requiring hospital admission: a Danish population-based cohort study. Lancet Infect Dis 2021:S14. Epub May 10, 2021. PMID:33984263

5. Al-Aly Z, Xie Y, Bowe B. High-dimensional characterization of post-acute sequelae of COVID-19. Nature 2021;594:259-64. PMID:33887749 https://doi.org/10.1038/s41586-021-03553-9

6. Jacobs LG, Gourna Paleoudis E, Lesky-Di Bari D, et al. Persistence of symptoms and quality of life at 35 days after hospitalization for COVID-19 infection. PLoS One 2020;15:e0243882. PMID:33306721 https://doi.org/10.1371/journal.pone.0243882

7. Havervall S, Rosell A, Phillipson M, et al. Symptoms and functional impairment assessed 8 months after mild COVID-19 among health care workers. JAMA 2021;325:2015-6. PMID:33825846 https://doi. org/10.1001/jama.2021.5612

8. Hernandez-Romieu AC, Leung S, Mbanya A, et al. Health care utilization and clinical characteristics of nonhospitalized adults in an integrated health care system 28-180 days after COVID-19 diagnosis-Georgia, May 2020-March 2021. MMWR Morb Mortal Wkly Rep 2021;70:644-50 PMID:33914727 https://doi.org/10.15585/mmwr.mm7017e3

9. Arnold DT, Milne A, Samms E, Stadon L, Maskell NA, Hamilton FW. Symptoms after COVID-19 vaccination in patients with persistent symptoms after acute infection: a case series. Ann Intern Med 2021. Epub May 25, 2021. PMID:34029484 https://doi.org/10.7326/M21-1976 